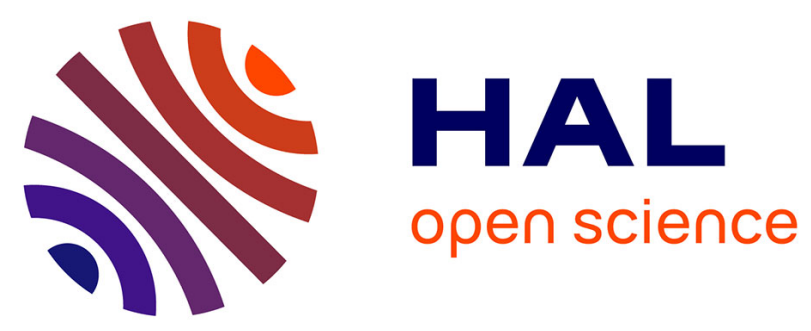

\title{
A dynamic model and an algorithm for short-term supply chain scheduling in the smart factory industry 4.0
}

Dmitry Ivanov, Alexandre Dolgui, Boris Sokolov, Frank Werner, Ivanova Marina

\section{- To cite this version:}

Dmitry Ivanov, Alexandre Dolgui, Boris Sokolov, Frank Werner, Ivanova Marina. A dynamic model and an algorithm for short-term supply chain scheduling in the smart factory industry 4.0. International Journal of Production Research, 2016, 54 (2), pp.386-402. 10.1080/00207543.2014.999958 . emse-01109312

\section{HAL Id: emse-01109312 \\ https://hal-emse.ccsd.cnrs.fr/emse-01109312}

Submitted on 15 Nov 2021

HAL is a multi-disciplinary open access archive for the deposit and dissemination of scientific research documents, whether they are published or not. The documents may come from teaching and research institutions in France or abroad, or from public or private research centers.
L'archive ouverte pluridisciplinaire HAL, est destinée au dépôt et à la diffusion de documents scientifiques de niveau recherche, publiés ou non, émanant des établissements d'enseignement et de recherche français ou étrangers, des laboratoires publics ou privés.

\section{다)(1) $(5$}

Distributed under a Creative Commons Attribution - NonCommerciall 4.0 International 


\title{
A dynamic model and an algorithm for short-term supply chain scheduling in the smart factory industry 4.0
}

\author{
Dmitry Ivanov $^{\mathrm{a} *}$, Alexandre Dolgui ${ }^{\mathrm{b}}$, Boris Sokolov $^{\mathrm{c}, \mathrm{f}}$, Frank Werner $^{\mathrm{d}}$ and Marina Ivanova ${ }^{\mathrm{e}}$ \\ ${ }^{a}$ Chair International Supply Chain Management, Berlin School of Economics and Law, Berlin, Germany; ${ }^{b}$ Ecole Nationale \\ Supérieure des Mines, FAYOL-EMSE, LIMOS, UMR 6158 CNRS, Saint-Etienne, France; ${ }^{c} I T M O$ University, Saint Petersburg, Russia, \\ ${ }^{d}$ Otto-von-Guericke University Magdeburg, Magdeburg, Germany; ${ }^{e}$ Faculty of Business Administration, Department of Industrial \\ Management, Chemnitz University of Technology, Chemnitz, Germany; ${ }^{f}$ Intelligent Control Systems Lab, SPIIRAS, Russia
}

Smart factories Industry 4.0 on the basis of collaborative cyber-physical systems represents a future form of industrial networks. Supply chains in such networks have dynamic structures which evolve over time. In these settings, short-term supply chain scheduling in smart factories Industry 4.0 is challenged by temporal machine structures, different processing speed at parallel machines and dynamic job arrivals. In this study, for the first time, a dynamic model and algorithm for short-term supply chain scheduling in smart factories Industry 4.0 is presented. The peculiarity of the considered problem is the simultaneous consideration of both machine structure selection and job assignments. The scheduling approach is based on a dynamic non-stationary interpretation of the execution of the jobs and a temporal decomposition of the scheduling problem. The algorithmic realisation is based on a modified form of the continuous maximum principle blended with mathematical optimisation. A detailed theoretical analysis of the temporal decomposition and computational complexity is performed. The optimality conditions as well as the structural properties of the model and the algorithm are investigated. Advantages and limitations of the proposed approach are discussed.

Keywords: supply chain scheduling; smart factory; structure dynamics; flexible flow shop; alternative machines; optimal programme control

\section{Introduction}

Most of the new factory concepts share attributes of smart networking (Dolgui and Proth 2010; Ivanov, Sokolov, and Pavlov 2013; Chick, Huchzermeier, and Netessine 2014; Davis et al. 2012; Villa, O'Brien, and Burlat 2012). That is why it becomes a timely and crucial topic to consider supply chains (SC) as collaborative cyber-physical systems (Camarinha-Matos and Macedo 2010; Zhuge 2011; Ivanov, Sokolov, and Dilou Raguinia 2014). Cyber-physical systems incorporate elements from both information and material subsystems which are integrated and decisions in them are cohesive (Zhuge 2011).

In addition, such systems evolve through adaptation and reconfiguration of their structures, that is through structure dynamics (Ivanov, Sokolov, and Kaeschel 2010; Ivanov, Sokolov, and Dolgui 2014; Ivanov and Sokolov 2012b). Smart factories Industry 4.0 on the basis of collaborative cyber-physical systems represents a future form of industrial networks. According to a PWC survey (PWC 2013), 50\% of German enterprises plan a networking and 20\% are already involved into a smart factory Industry 4.0. Industry 4.0 represents a smart manufacturing networking concept where machines and products interact with each other without human control. SCs in such networks have dynamic structures which evolve over time. In these settings, short-term SC scheduling in smart factories Industry 4.0 is challenged by temporal machine structures, different processing speed at parallel machines and dynamic job arrivals. In terms of scheduling theory, scheduling in smart factory Industry 4.0 can be classified as flexible job or flowshop (Pinedo 2008; Rajabinasab and Mansour 2011; Sawik 2012; Na and Park 2013). This results in a dynamic scheduling environment with structure dynamics. We regard this domain as an opportunity for research and development.

In scheduling theory, considerable achievements can be stated regarding assignment and sequencing problems in the context of multi-stage systems (Blazewicz et al. 2001; Lauff and Werner 2004). Most papers on multi-stage systems deal with problems, where the computational complexity represents the most critical challenge (Chiou et al. 2012). Another challenge is the different processing speed at the machines which influence the task times (Kyparisis and Koulamas 2006).

*Corresponding author. Email: divanov@hwr-berlin.de 
In this study, we study a multi-objective, multi-stage flexible flow-shop scheduling problem with alternative machines at each stage with different time-dependent processing speed, time-dependent machine availability and ordered jobs where job splitting is allowed. Examples of such problems can be found in the studies by Kyparisis and Koulamas (2006) and Tahar et al. (2006) and Bożek and Wysocki (2015). The peculiarity of the problem under consideration is the simultaneous consideration of both machine structure selection, continuous flows, and job assignment. In these settings, an interesting constellation of discrete and continuous elements can be observed. On the one hand, an assignment problem is discrete by nature and requires the introduction of binary variables, that is discrete optimisation techniques can be correctly used here. At the same time, a non-stationary job execution can be correctly described in terms of continuous optimisation (Shah and Ierapetritou 2012; Subramanian et al. 2013). An additional peculiarity of such simultaneous consideration is that both the machine structures and the flow parameters may be uncertain and change in dynamics and are, therefore, non-stationary.

The remainder of this study is organised as follows. Section 2 is devoted to a literature review. Section 3 presents the methodology and problem statement. In Section 4, the model is described and a scheduling problem in terms of OPC is stated. Section 5 presents the algorithm and analyses the computational procedure. In Section 6, the optimality, existence and complexity are analysed. The study concludes by discussing the main findings.

\section{Literature review}

Beginning with the work of Johnson (1954), flow-shop scheduling models (i.e. models where a set of jobs must be processed on a number of machines sequentially disposed and the operations for different jobs have to be processed in the same order) have been extensively considered in the literature, including, for instance, Gupta, Neppalli, and Werner (2001), Kubzin, Potts, and Strusevich (2009), Dugardin, Yalaoui, and Amodeo (2010), Ribas, Leisten, and Framiñan (2010). Since the problems of this class, with a few exceptions, have been proved to be NP-hard (Gonzalez and Sahni 1978), heuristic solutions are predominantly applied in practice (Jungwattanakit et al. 2008, 2009; Laha and Sarin 2009; Martin 2009; Werner 2013).

Scheduling with alternative parallel machines has also been a large research avenue over the past few decades. In such systems, the goal is to optimise both the selection of machines for each part and the loading sequences of the parts to the machines to improve the productivity (Hankins, Wvsk, and Fox 1984) or to minimise the makespan (Weglarz 1976). Blazewicz, Dror, and Weglarz (1991) review showed that these problems are NP-hard. Józefowska et al. (2002) presented a heuristic approach to allocating a continuous resource in discrete-continuous scheduling problems to minimise the makespan. In some cases, for example for identical processors with unit processing times, such a scheduling problem can be transformed to a transportation problem and solved with integer programming (IP) methods (Graham et al. 1979). Kyparisis and Koulamas (2006) considered a multi-stage flexible flow-shop scheduling problem with uniform parallel machines at each stage and makespan minimisation. This study proposed a heuristic schedule computation for this strongly NP-hard problem. Tahar et al. (2006) considered the problem of scheduling a set of independent jobs with sequence-dependent setup times and job splitting on a set of identical parallel machines such that the maximum completion time (makespan) is minimised. For this NP-hard problem, this study developed a heuristic algorithm using linear programming (LP).

Consideration has also been given to continuous flows which are typical for the processing industry, for example petrochemistry, energy supply, oil and gas industries (Mujawar, Huang, and Nagi 2012; Subramanian et al. 2013). A practical challenge is that at each stage, alternative machines may perform the operations. This creates flexibility in the process plan and requires both a machine assignment and sequencing the tasks (Yu et al. 2011). The optimiszation objectives in practice are multiple ones and may be related to the maximisation of the processed jobs, completing the jobs on time (e.g. minimisation of maximal lateness) and an equal machine utilisation over time.

Bożek and Wysocki (2015) analysed continuous flow flexible job shop (CF-FJS) problem that combines the flexible job shop (FJS) problem and a dedicated continuous material flow model (MFM). In this study, the operations are represented by material flow functions derived by integration of arbitrarily defined speed patterns. Variable speed of processing and continuous material flow lead to position-dependent processing times and overlapping in operations. A tabu search scheduling algorithm is proposed to solve the model.

Let us turn to the OPC applications. The studies by Holt et al. (1960), Hwang, Fan, and Erikson (1967), Zimin and Ivanilov (1971) and Moiseev (1974) were among the first to apply the OPC and the maximum principle to multi-level and multi-period master production scheduling that determined the production as an optimal control with a corresponding trajectory of the state variables (i.e. the inventory). This stream was continued by Kimemia and Gershwin (1983), who applied a hierarchical method in designing a solution procedure to the overall model, and by Khmelnitsky, Kogan, and Maimom (1997) for planning continuous-time flows in flexible manufacturing.

The managers are always interested in non-deterministic approaches to scheduling where scheduling is interconnected to the control function (Maccarthy and Liu 1993). The studies by Sarimveis et al. (2008) and Harjunkoski et al. 
(2014) showed a wide range of advantages regarding the application of control-theoretic models in combination with other techniques to production and logistics. They include, first of all, a non-stationary process view and accuracy of continuous time. In addition, a wide range of analysis tools from control theory regarding stability, controllability, adaptability, etc. may be used if a schedule is described in terms of control. However, the calculation of the OPC with direct methods of the continuous maximum principle has not been proved to be efficient (Ivanov and Sokolov 2012a). So the application of OPC to scheduling is not a trivial problem for two reasons. First, a conceptual problem consists of the continuous values of the control variables. Second, a computational problem with a direct method of the maximum principle exists. These shortcomings set limitations on the application of OPC to purely combinatorial problems.

\section{Problem statement and methodical approach}

\subsection{Problem statement}

Consider the problem statement subject to the following list of notations:

\section{Sets}

$\bar{B}=\left\{\bar{B}^{(i)}, i \in \bar{N}, \bar{N}=(1, \ldots, \bar{n})\right\} \quad$ is the set of jobs

$D=\left\{D_{\mu}^{i}, \mu \in \bar{S}, \bar{S}=\left(1, \ldots, s_{i}\right)\right\} \quad$ is the set of operations

$M=\left\{M^{j}, j \in N, N=(1, \ldots, n)\right\} \quad$ is the set of machines

$\Gamma \quad$ is the set of precedence operations

$\mathbf{Q}(\mathbf{x}(t)) \quad$ is the domain of feasible control inputs

$\widetilde{\mathbf{Q}}(\mathbf{x}(t)) \quad$ is the extended domain of feasible control inputs

$\underset{\tilde{K}}{\tilde{K}} \quad$ is the initial class of feasible control inputs

$\tilde{\tilde{K}} \quad$ is the extended class of feasible control inputs

\section{Parameters}

$\underset{\tilde{R}}{a} \quad$ is the planned processing volume

$\tilde{\tilde{R}} \quad$ is the total machine capacity

$T_{0} \quad$ is the start instant of time of the scheduling horizon

$T_{f} \quad$ is the end instant of time of the scheduling horizon

$c \quad$ is the processing intensity

$\xi(t) \quad$ is the vector of perturbation impacts

$\Delta t \quad$ is the step length of integration for the main and the conjugate system

$\mathbf{h}_{0}^{(o)}, \mathbf{h}_{1}^{(o)} \quad$ are known differentiable functions that determine the end conditions of the vector

\section{Indices}

$i \quad$ is the job index

$j \quad$ is the machine index

$\mu \quad$ is the operation index (i.e. number of the operation in the job)

$l \quad$ is the index of the flow-shop stage

$o \quad$ is the index of parameters and variables in the model $\mathbf{M}_{o}$

$k \quad$ is the index of parameters and variables in the model $\mathbf{M}_{\boldsymbol{k}}$

$f \quad$ is the index of parameters and variables in the model $\mathbf{M}_{\boldsymbol{f}}$

$z \quad$ is the number of the Hamiltonian function

$r \quad$ is the number of the iteration of the algorithm

\section{Continuous variables and functions}

is the current time instant

$\sigma \quad$ is the duration of the planning interval

$x^{(o)} \quad$ is a variable characterising the state of an operation, where $(o)$ indicates the relationship of the state variable $x$ to the operation states

$\varepsilon(t) \quad$ is the given preset matrix time function of time-spatial constraints 


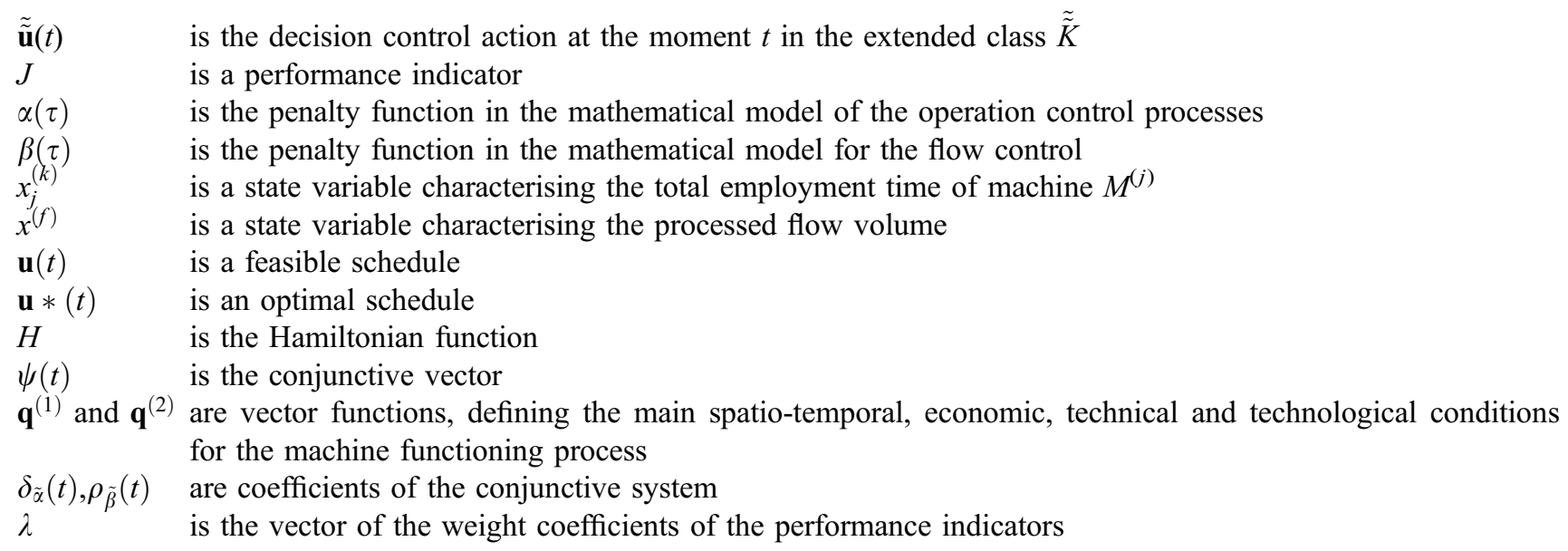

\section{Discrete variables}

$u^{(o)}(t) \quad$ is the decision control action at the moment $t$

$\overline{\bar{m}}_{1} \quad$ is the maximal dimensionality of the assignment problem

$\tilde{\tilde{n}} \quad$ is the maximal number of parallel executed jobs within the planning interval

\section{Others}

$\mathbf{M}_{o}$

$\mathbf{M}_{\boldsymbol{k}}$

$\mathbf{M}_{f}$

M

is an optimal programme control problem

$\Lambda \quad$ is the relaxed problem

$\mathrm{R} \quad$ is an operator in the optimal control algorithm

At each $l$-stage, some uniform alternative machines $M^{(j)}$ from the set $M=\left\{M^{(j)}, j \in N, N=(1, \ldots, n)\right\}$ exist. At each $l$-stage, some uniform alternative machines $M^{(j)}$ exist. Consider the operations $D_{\mu}^{(i)}$ from the set $D=\left\{D_{\mu}^{(i)}, \mu \in \bar{S}, \bar{S}=\left(1, \ldots, s_{i}\right)\right\}$, each of which belongs to a job $\bar{B}^{(i)}$ from the set $\bar{B}=\left\{\bar{B}^{(i)}, i \in \bar{N}, \bar{N}=(1, \ldots, \bar{n})\right\}$. The independent jobs consist of a chain of operations. All jobs are assumed to be available for processing at time 0 . Each machine $M^{(j, l)}$ is capable of producing all the operations at the $l$-stage, but it can handle only one job at a time. Note that for a simplification, the stage index $l$ is omitted in the further progress of this study, and it is assumed to be considered in the machine indexes $j$ subject to the non-stationary machine availability at each stage (i.e. 'availability windows'), which is expressed in the preset matrix time function $\varepsilon_{i j}(t)$. The structure considered is presented in Figure 1.

At each of the stages, each machine $M^{(j)}$ has a speed (i.e. an effective processing intensity) $c_{i \mu j}$ that is subject to the total machine capacity $\tilde{R}_{j}$. The impact of the speed $c_{i \mu j}(t)$ is that the machine $M^{(j)}$ can process $a_{i \mu j}$ units subject to the

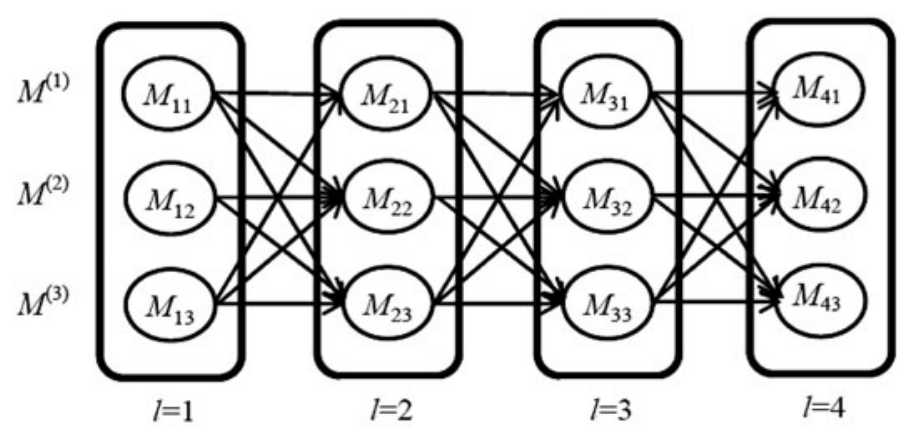

Figure 1. Problem structure. 
planned processing volume $a_{i \mu}$ and $c_{i \mu j}(t)$. An operation $D_{\mu}^{(i)}$ may start only after the previous operation $D_{\mu-1}^{(i)}$ has been completed. All jobs have to be completed by time $T_{f}$. For a simplification of the model and the algorithm presentation in this study, we allow pre-emptions and do not consider setups (i.e. in Figure 1, the operations may be partially executed on different machines at each of the stages as known from the job splitting literature, e.g. Tahar et al. 2006).

The problem consists of scheduling the operations taking into account flow dynamics control subject to three objectives: $J_{1}$ - minimisation of total lateness (subject to $T_{f}$ ), $J_{2}$ - maximisation of the volume of the fully completed jobs (subject to $a_{i \mu}$ and $a_{i \mu j}$; i.e. in the ideal case $a_{i \mu}=a_{i \mu j}$ for all jobs subject to $c_{i \mu j}(t)$ and $\varepsilon_{i j}(t)$; a strong requirement on the full completion of all jobs by the time $T_{f}$ may also be included) and $J_{3}$ - equal utilisation of the machines (subject to $\tilde{\tilde{R}}_{j}$ ).

\subsection{Methodical approach}

The underlying theory for studying multi-stage, multi-period dynamic systems with continuous variables and performance indicators accumulated over time is optimal control theory in general and optimal programme control (OPC) in particular (Athaus and Falb 1966; Lee and Markus 1967; Sethi and Thompson 2000). At the same time, a direct application of OPC to a pure combinatorial problem is questionable.

The basic technical idea of our approach is that the elements of OPC can extend the existing MP scheduling techniques. For this reason, we do not use OPC for solving the combinatorial problem but rather to enhance the existing MP algorithms regarding the non-stationarity, flow control and continuous material flows. If the control variables are presented as binary variables, it might become possible to incorporate them into the assignment problem. We apply methods of discrete optimisation to combinatorial tasks within certain time intervals and to use the OPC with all its advantages (i.e. accuracy of continuous time, integration of planning and control, and the operation execution parameters as time functions) for (1) the flow control within the operations and (2) interlinking the decomposed solutions.

The basic computational idea of this approach is the fact that the operation execution and machine availability are dynamically distributed in time over the planning horizon. As such, not all operations and machines are involved in the decision-making at the same time. Therefore, it becomes quite natural to transit from large-size allocation matrices with a high number of binary variables to a scheduling problem that is dynamically decomposed. Following an approach to decompose the solution space and to use exact methods over its restricted sub-spaces, we propose to use the OPC theory for the dynamic decomposition of the scheduling problem. OPC is mainly used for the dynamic decomposition but not for the calculations. The computational procedure is transferred to the MP methods (Ivanov, Sokolov, and Pavlov 2013, 2014). The solution at each time point is calculated with MP. OPC is used for modelling the execution of the operations and interlinking the MP solutions over the planning horizon. Hence, the solution procedure becomes independent of the continuous optimisation algorithms and can be of discrete nature, for example an integer assignment model. The maximum principle guarantees that the optimal solutions of the instantaneous problems give an optimal solution to the overall problem (Pontryagin et al. 1964; Boltyanskiy 1973; Sethi and Thompson 2000).

The original dynamic interpretation of the assignment of the operations to a non-stationary set of machines can be exemplified in the following way. Consider four machines and six jobs $\bar{B}^{(i)}$, each of which is composed of 3-6 operations $D_{\mu}^{(i)}$. At each time instant, only one operation can be processed on one machine (see Figures 2-5).

Note that in this example, for a simplification, we consider only a one-stage system with four alternative uniform machines. In the model, a multi-stage flow shop will be considered as shown in Figure 1. In Figures 2-5, the execution

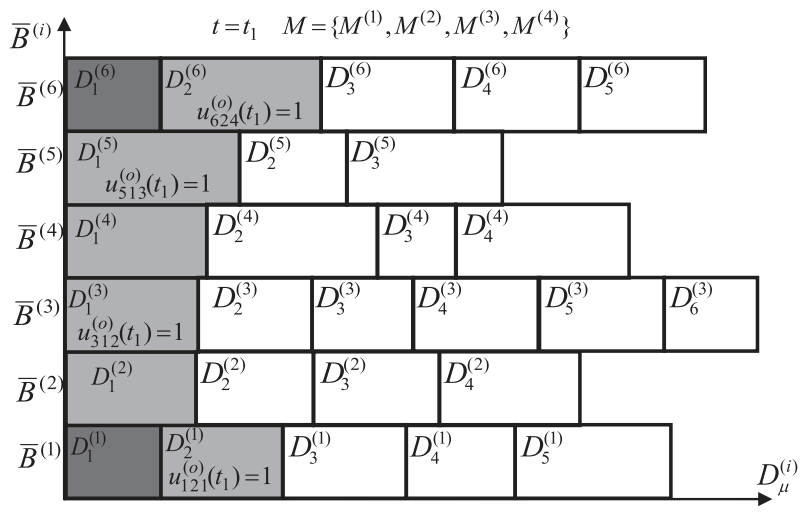

Figure 2. Dynamic representation of the scheduling model. 


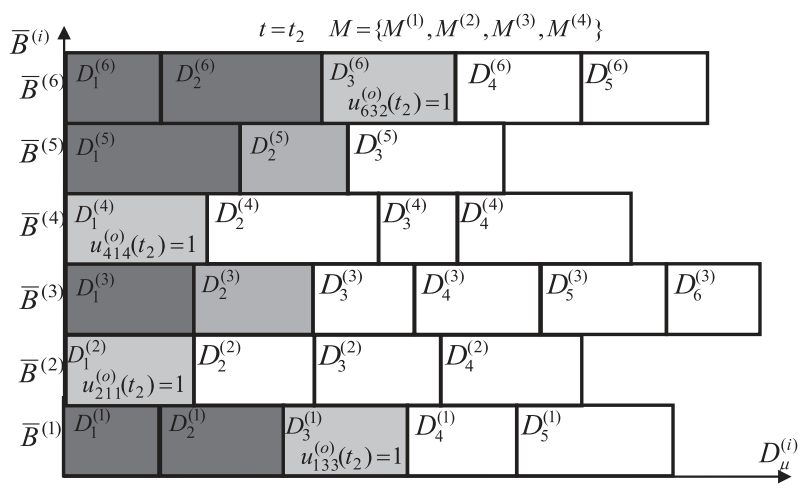

Figure 3. Dynamic representation of the scheduling model.

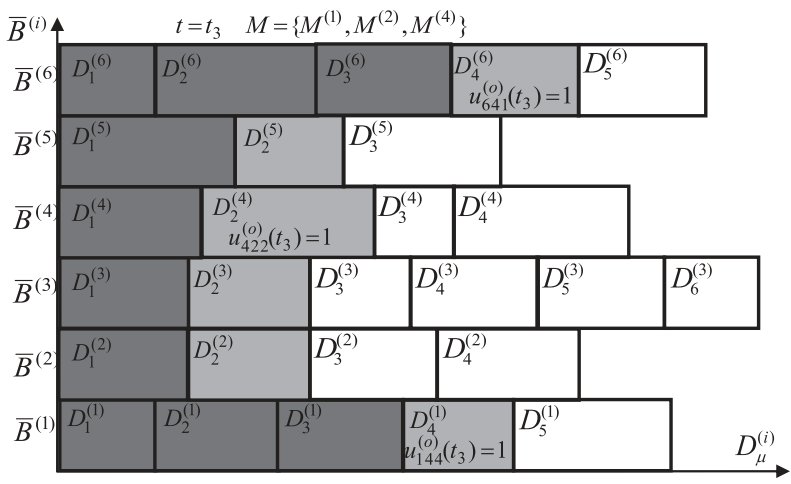

Figure 4. Dynamic representation of the scheduling model.

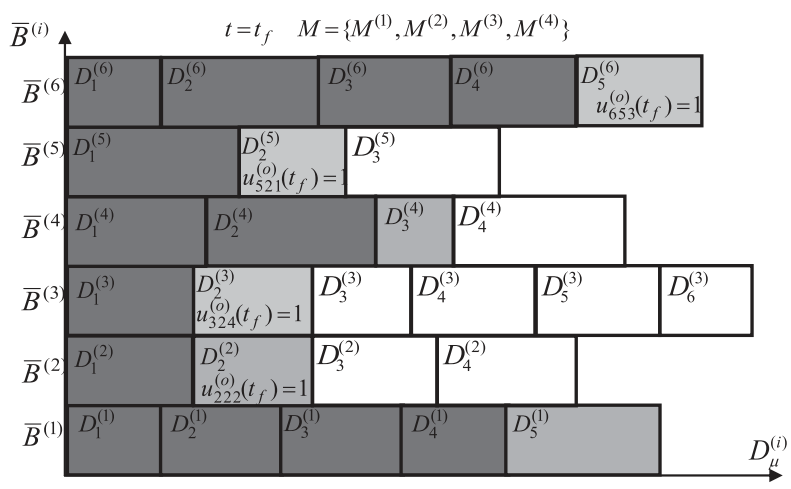

Figure 5. Dynamic representation of the scheduling model.

dynamics of six jobs is presented for four time instants $t=t_{1}, t_{2}, t_{3}, t_{f}$. Note that in this example of a continuous problem statement, $t=t_{1}, t_{2}, t_{3}, t_{f}$ corresponds to $l=1,2,3,4$ in the discrete case as presented in Figure 1 . Different colours describe current execution states. The operations marked in black have already been completed. The operations marked in grey may be executed subject to the machine availability and precedence relations. The operations marked in white cannot be executed yet because of the precedence relations. For example, at $t=t_{2}$, the operation $D_{2}^{(4)}$ cannot be assigned since the operation $D_{1}^{(4)}$ is still being processed with the use of the machine $M^{(4)}\left(u_{i \mu j}^{(o)}\left(t_{2}\right)=1\right)$.

From Figures $2-5$, it can be observed that at each time instant, the assignment decisions consider only the grey coloured operations subject to some available ('competing') machines, that is the large-scale multi-dimensional combinatorial matrix is decomposed. The assignment of a machine $M^{(j)}$ to the execution of the operation $D_{\mu}^{(i)}$ can be described by the piecewise continuous function $u_{i \mu j}^{(o)}(t)$ that becomes equal to 1 in the case of an assignment. In the 
following course of this study (see Section 4), these functions will play the role of OPC within the proposed dynamic model of the execution of the operations.

In the upper part of Figures 2-5, the machines $M^{(j)}$ are listed which are available at the current time instant. At $t=t_{1}, t_{2}, t_{f}$, the four machines $M^{(1)}, M^{(2)}, M^{(3)}, M^{(4)}$ are available. At $t=t_{3}$, only the three machines $M^{(1)}, M^{(2)}, M^{(4)}$ are available. This non-stationary set of machine availability will be represented in the model (see Section 5) with the help of the preset matrix time function $\varepsilon_{i j}(t)$.

It can be observed from Figures 2-5 that the current dimensionality of the considered scheduling problem for $t=t_{1}, t_{2}, t_{3}, t_{f}$ is determined by the dimensionality of the grey coloured area. The operations in the black and white areas are not considered at the given time points and, therefore, will not influence the mathematical model for the assignment.

\section{Mathematical model}

The formal statement of the scheduling problem will be produced, as it has been noted above, via a dynamic interpretation of the execution processes of the operations. In the remainder of this section, we will consider the partial dynamic models in more detail.

\subsection{Mathematical model for the control processes of the operations (model $M_{0}$ )}

Let us consider the mathematical model for processing the operation $D_{\mu}^{(i)}$ in the job $\bar{B}^{(i)}$. The following notations can be introduced:

$x_{i \mu}^{(o)}$ is a variable characterising the state of the operation $D_{\mu}^{(i)}$, where $(o)$ indicates the relationship of the state variable $x$ to the operation states.

$\varepsilon_{i j}(t)$ is the given preset matrix time function of the time-spatial constraints.

$u_{i \mu j}^{(o)}(t)$ is the decision control action at the moment $t$.

$t$ is the current time instant; $t \in T=\left(T_{0}, T_{f}\right]$ is the planning horizon.

$T_{0}\left(T_{f}\right)$ is the start and end time instant of the planning horizon.

The processing dynamics of the operation $D_{\mu}^{(i)}$ can be expressed as follows:

$$
\frac{d x_{i \mu}^{(o)}}{d t}=\dot{x}_{i \mu}^{(o)}=\sum_{j=1}^{n} \varepsilon_{i j}(t) u_{i \mu j}^{(o)}(t) .
$$

Equation (1) represents the operation execution dynamics in which the non-stationarity of the execution of the operations is reflected. We have $\varepsilon_{i j}(t)=1$, if machine $M^{(j)}$ is available, and $\varepsilon_{i j}(t)=0$, otherwise (e.g. a constraint on the production shift from $7 \mathrm{am}$ to $4 \mathrm{pm}) . u_{i \mu j}^{(o)}(t)$ is a decision variable. We have $u_{i \mu j}^{(o)}(t)=1$ at the time point $t$, if the operation $D_{\mu}^{(i)}$ is assigned to the machine $M^{(j)}$, and $u_{i \mu j}^{(o)}(t)=0$, otherwise.

The continuous time allows to represent the execution of the operations at each time point and, therefore, to obtain additional information about the execution of the operations. An example of a control profile for the execution of the operations is given in Figure 6.

In Figure 6, an example of a control profile for the execution of one operation on one machine is presented. The usage of continuous time allows a non-stationary analysis of the schedule execution (e.g. the machine availability

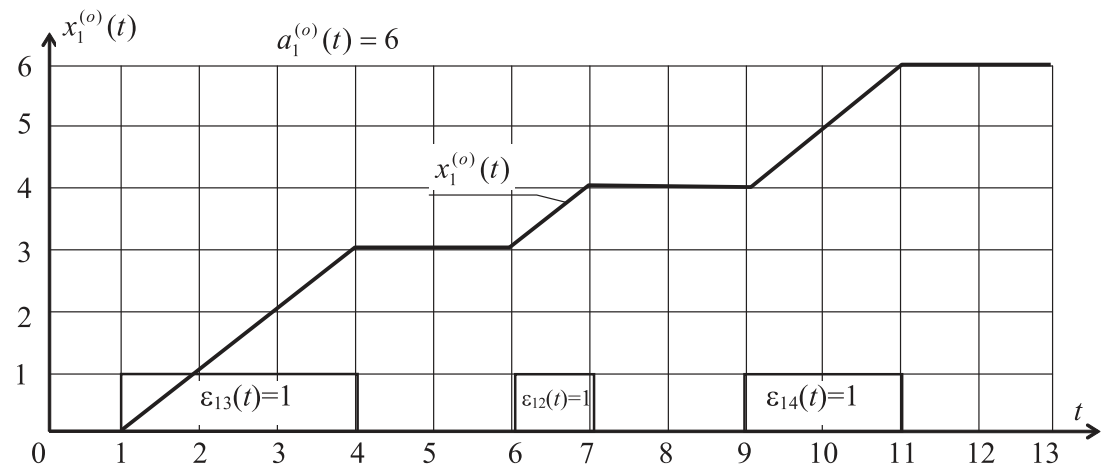

Figure 6. Example of the execution dynamics and an assignment decision profile. 
between 8:00 and 10:00 am or between 9:00 and 10:00 am may have a different impact on the schedule performance). The state variable $x(t)$ accumulates the executed (processed) volume of the considered operation. Assuming that the planned execution volume is 6 units, it can be observed from Figure 6 that the given operation can be fully executed (i.e. $a_{i \mu}^{(o)}=x_{i \mu}^{(o)}$ ) on the given machine and completed on time (by $T_{f}$ ) with a processing time of 13 time units. In this case, the control variable $u_{i \mu j}^{(o)}(t)$ (Equation 1) will switch to 1, which means that the assignment is possible. Such principles are equivalent to those used in the further models.

The control actions are constrained as follows:

$$
\begin{gathered}
\sum_{i=1}^{\bar{n}} \sum_{\mu=1}^{s_{i}} u_{i \mu j}^{(o)}(t) \leq 1, j=1, \ldots, n ; \sum_{j=1}^{n} u_{i \mu j}^{(o)}(t) \leq 1, i=1, \ldots, \bar{n}, \mu=1, \ldots, s_{i}, \\
\sum_{j=1}^{n} u_{i \mu j}^{(o)}\left[\sum_{\alpha \in \Gamma_{i \mu_{1}}^{-}}\left(a_{i \alpha}^{(o)}-x_{i \alpha}^{(o)}\right)+\prod_{\beta \in \Gamma_{i \mu_{2}}^{-}}\left(a_{i \beta}^{(o)}-x_{i \beta}^{(o)}\right)\right]=0, \\
u_{i \mu j}^{(o)}(t) \in\{0,1\},
\end{gathered}
$$

where conditions (2)-(4) hold for $i=1, \ldots, n, \mu=1, \ldots, s_{i}, j=1, \ldots, \bar{n} ; \Gamma_{i \mu_{1}}^{-}, \Gamma_{i \mu_{2}}^{-}$are the sets of precedence operations regarding the operation $D_{\mu}^{(i)}, \sum_{j=1}^{n} u_{i \mu_{j}}^{(o)} \sum_{\alpha \in \Gamma_{i \mu_{1}}^{-}}\left(a_{i \alpha}^{(o)}-x_{i \alpha}^{(o)}(t)\right)=0$ is an 'and' constraint, which denotes the condition of the total processing of all the predecessor operations, $\sum_{j=1}^{n} u_{i \mu_{j}}^{(o)} \prod_{\beta \in \Gamma_{i \mu_{2}}^{-}}\left(a_{i \beta}^{(o)}-x_{i \beta}^{(o)}\right)=0$ is an 'or' constraint, which denotes the condition of the processing of at least one of the predecessor operations.

Constraints (2) define the $1 \times 1$ assignment problem. Constraints (3) bring the natural time logic into the model and determine the precedence relations by blocking the operation $D_{\mu}^{(i)}$ until the previous operations $D_{\alpha}^{(i)}, D_{\beta}^{(i)}$ have been completed.

In order to assess the results of the execution of the operations, we define the following start and end conditions:

$$
\mathbf{h}_{0}^{(o)}\left(\mathbf{x}^{(o)}\left(T_{0}\right)\right) \leq \mathbf{0} ; \quad \mathbf{h}_{1}^{(o)}\left(\mathbf{x}^{(o)}\left(T_{f}\right)\right) \leq \mathbf{0},
$$

where $\mathbf{h}_{0}^{(o)}, \mathbf{h}_{1}^{(o)}$ are known differentiable functions that determine the start and end conditions of the vector

$$
\mathbf{x}^{(o)}=\left(\mathbf{x}_{11}^{(o) \mathrm{T}}, \ldots, \mathbf{x}_{\bar{n} s_{i}}^{(o)}\right)^{\mathrm{T}} .
$$

The initial and end conditions (7) and (8) specify the values of the variables at the beginning and end of the planning period, namely:

at the moment

$$
t=T_{0}: x_{i \mu}^{(o)}\left(T_{0}\right)=0
$$

at the moment

$$
t=T_{f} x_{i \mu}^{(o)}\left(T_{f}\right)=a_{i \mu}^{(o)}
$$

The OPC $\mathbf{u}(t)$ and the state trajectory $\underline{\mathbf{x}}=\mathbf{f}(\mathbf{x}, \mathbf{u}, t)$ should be determined so that the constraints (7) and (8) are met; in other words, the desired values of the performance indicators should be achieved as an analogy to goal programming.

Constraint (7) reflects that, at the beginning, the volume of the executed operations is equal to zero (in the case that a certain volume of the orders is to be transferred from the previous planning period to the beginning of the current planning period, this should be reflected in (8)). Condition (8) reflects the desired end state, that is the completion of the operations by the time $T_{f}$.

According to the problem statement, let us introduce the following performance indicators (objectives):

$$
\begin{gathered}
J_{1}^{(o)}=\frac{1}{2} \sum_{i=1}^{\bar{n}} \sum_{\mu=1}^{s_{i}}\left(a_{i \mu}^{(o)}-x_{i \mu}^{(o)}\left(T_{f}\right)\right)^{2}, \\
J_{2}^{(o)}=\sum_{i=1}^{\bar{n}} \sum_{\mu=1}^{s_{i}} \sum_{j=1}^{n} \int_{T_{0}}^{T_{f}} \alpha_{i \mu}^{(o)}(\tau) u_{i \mu j}^{(o)}(\tau) d \tau .
\end{gathered}
$$


The performance indicator $J_{1}^{(o)}$ (function (9)) characterises the accuracy of the accomplishment of the end conditions, that is the volume of the completed operations by the time $T_{f}$. This can also express the extent of losses caused by a non-fulfilment of the end conditions. The objective function (10) minimises total maximum lateness using penalties. The function $\alpha_{i \mu}^{(o)}(\tau)$ is assumed to be known for each operation.

\subsection{Mathematical model for the machine control (model $M_{\mathrm{k}}$ )}

Let us introduce Equation (11) to assess the total machine availability time:

$$
\frac{d x_{j}^{(k)}}{d t}=\dot{x}_{j}^{(k)}=\sum_{i=1}^{\bar{n}} \sum_{\mu=1}^{s_{i}} u_{i \mu j}^{(o)}(t),
$$

where $(k)$ indicates the relationship of the state variable $x$ to the machines. Equation (11) represents the machine utilisation. The variable $x_{j}^{(k)}$ characterises the total employment time of machine $M^{(j)}$. Equation (11) corresponds to Equation (1) subject to the control variable $u_{i \mu j}^{(o)}(t)$. We have $u_{i \mu j}^{(o)}(t)=1$ at the time point $t$, if the machine $M^{(j)}$ is occupied by the operation $D_{\mu}^{(i)}$ and $u_{i \mu j}^{(o)}(t)=0$ otherwise.

The end conditions are identical to Equations (5)-(8).

According to the problem statement, let us introduce the following performance indicator:

$$
J_{1}^{(k)}=\frac{1}{2} \sum_{j=1}^{n}\left(T-x_{j}^{(k)}\left(T_{f}\right)\right)^{2} .
$$

The indicator $J_{1}^{(k)}$ helps to estimate the uniformity of the machine use at the end point $t=T_{f}$ of the planning period. For example, in the supply chain scheduling context, this may be a requirement for a supply chain collaboration.

\subsection{Mathematical model for the flow control (model $M_{\mathrm{f}}$ )}

The interrelations and mutual impacts of the assignment and flow control still remain an open research question. In the proposed approach, these decisions are considered simultaneously. Recall that the task times may differ regarding different speeds $c_{i \mu j}(t)$ and machine availabilities $\varepsilon_{i j}(t)$. For instance, the assignment of an operation from Figure 6 to another machine could result in a different execution control profile and task time. For this reason, the assignments from the model $\mathbf{M}_{\boldsymbol{o}}$ (made on the basis of the volumes $a_{i \mu}$ ) are now subject to further optimisation regarding the flow dynamics control.

An assignment of an operation to a machine and the starting execution of the operations cause dynamic flows of the processed products. Let us introduce a model for the flow dynamics control (13):

$$
\frac{d x_{i \mu j}^{(f)}}{d t}=\dot{x}_{i \mu j}^{(f)}=u_{i \mu j}^{(f)}
$$

where $(f)$ indicates the relationship of the state variable $x$ to the flows. Equation (12) corresponds to Equations (11) and (13). The economic sense of Equation (13) consists in the dynamic representation of the material flows resulting from the execution of the operations on the machine $M^{(j)}$. The meaning of Equation (13) is very close to a system dynamics model to balance the flows in a system. However, the proposed approach also considers the strictly defined logic of the execution of the operations (model $\mathbf{M}_{\boldsymbol{o}}$ ). Moreover, the models of operations and flow control are interlinked linearly by Equation (14) and the conjunctive system (27)-(29).

In contrast to model $\mathbf{M}_{\boldsymbol{o}}$, the control variable $u_{i \mu j}^{(f)}(t)$ is not a binary variable, but is equal to the processed flow volume $x_{i \mu j}^{(f)}$ at each time point $t$. The model $\mathbf{M}_{f}$ uses the assignment results from the model $\mathbf{M}_{o}$ in the form of the control variables $u_{i \mu j}^{(o)}(t)$ and extends them by the actual processing speed of the machines subject to the following constraints:

$$
\begin{gathered}
0 \leq u_{i \mu j}^{(f)}(t) \leq c_{i \mu j}^{(f)} \cdot u_{i \mu j}^{(o)}, \\
\sum_{i=1}^{\bar{n}} \sum_{\mu=1}^{s_{i}} u_{i \mu j}^{(f)}(t) \leq \tilde{\tilde{R}}_{j}^{(f)} \cdot \xi^{(f)}(t) .
\end{gathered}
$$

Inequalities (14) use the assignment decisions $\left(u_{i \mu j}^{(o)}(t)\right)$ from the model $\mathbf{M}_{\boldsymbol{o}}$ and the processing speed $c_{i \mu j}(t)$ of the machines $M^{(j)}$ for the optimisation problems (13)-(18).

Constraints (15) reflect that the processing speed is constrained by $\tilde{\tilde{R}}_{j}^{(f)}$ taking into account the lower and upper bounds of some perturbation impacts $0 \leq \xi^{(f)}(t) \leq 1$ which may decrease the capacity availability. 
Remark: Equations (15) and (16) set up the attainable set (AS) of the OPC in a dynamic system, that is all possible states of the schedule execution subject to different variations of the parameters (e.g. the capacity availability). The introduction of Equation (15) allows to analyse feasible schedule executions under conditions of non-stationary perturbations. If so, an AS can be used to analyse the schedule robustness that is an ability to continue the schedule execution subject to the specified objectives in the presence of perturbations.

The end conditions in $\mathbf{M}_{\boldsymbol{f}}$ are identical to (5)-(8).

According to the problem statement, let us introduce the following objectives:

$$
\begin{gathered}
J_{1}^{(f)}=\frac{1}{2} \sum_{i=1}^{\bar{n}} \sum_{\mu=1}^{s_{i}} \sum_{j=1}^{n}\left(a_{i \mu j}^{(f)}-x_{i \mu}^{(f)}\left(T_{f}\right)\right)^{2}, \\
J_{2}^{(f)}=\frac{1}{2} \sum_{i=1}^{\bar{n}} \sum_{\mu=1}^{s_{i}} \sum_{j=1}^{n} \int_{T_{0}}^{T_{f}} \beta_{i \mu}^{(f)}(\tau) u_{i \mu j}^{(f)}(\tau) d \tau .
\end{gathered}
$$

The economic meaning of the objectives $(16)-(17)$ is identical to the objectives (9)-(10). The function $\beta_{i \mu}^{(f)}(\tau)$ is assumed to be known for each operation.

Remark 2. Note that the constraints (2)-(4) and (14)-(15) are identical to those in the MP models. However, at each $t$-point of time, the number of variables in the calculation procedure is determined by the operations, which are currently in the 'active zone' of scheduling, that is the operations marked in grey in Figures 2-5. For the problem sizes subject to the 'active zone', known methods for the solution of the MP models (e.g. the Hungarian method for $\mathbf{M}_{\boldsymbol{o}}$ or linear programming (LP) for $\mathbf{M}_{f}$ ) can be applied to the problem (1)-(17).

\subsection{Formulation of the scheduling problem}

The models described above can be presented in an integrated form (model $\mathbf{M}$ ). As mentioned above, the model should provide the decision-makers with alternatives to handle. The performance indicators may be weighted in different proportions depending on the planning goals and operational strategies. The preference relations (minmax, maxmin, etc.) form the Pareto space and allow the calculation of a general relative quality index (QI) (18) within the corresponding schedule $\mathbf{u}(t)$.

$$
\min \mathbf{J}(\mathbf{x}(t), \mathbf{u}(t), \xi(t), t)=\left\|\left(J_{1}^{(o)}, J_{2}^{(o)}, J_{1}^{(k)}, J_{1}^{(f)}, J_{2}^{(f)}\right)^{\mathrm{T}}\right\|
$$

where $J_{1}^{(o)}, J_{2}^{(o)}, J_{1}^{(k)}, J_{1}^{(f)}, J_{2}^{(f)}$ are the values of the performance indicators.

For a simplification, it is assumed that the transition from the vector form $\mathbf{J}$ to a scalar form $J_{G}$ has been performed on the basis of the weight coefficients $\lambda_{1}^{(o)}, \lambda_{2}^{(o)}, \lambda_{3}^{(k)}, \lambda_{4}^{(f)}, \lambda_{5}^{(f)}$.

Now, the scheduling problem can be formulated as the following problem of dynamic system control. The task is to find a feasible control $\mathbf{u}(t),\left[T_{0}, T_{f}\right)$ which ensures that the dynamic control model meets the constraint functions and guides the dynamic system (i.e. the schedule) $\dot{\mathbf{x}}=\mathbf{f}(t, \mathbf{x}, \mathbf{u})$ from the initial state to the specified final state subject to given end conditions and the uncertainty area under the disturbances $\xi(t)$. If there are several feasible controls (schedules), then the best one (optimal) should be selected in order to maximise (minimise) the components of $J_{G}$. We refer to this problem as $P S$.

\section{Computational procedure and analysis of the algorithm}

The computational procedure for the developed model is based on the integration of the main and conjunctive equation systems subject to the maximisation of the following Hamiltonian (19)-(21):

$$
\begin{gathered}
H\left(\mathbf{x}^{*}(t), \mathbf{u}^{*}(t), \psi(t)^{*}\right)=\max _{\tilde{\tilde{\mathbf{u}}} \tilde{\tilde{\mathbf{Q}}}(\mathbf{x})} \sum_{z=1}^{2} H_{l}(\mathbf{x}(t), \mathbf{u}(t), \psi(t)), \\
H_{1}=\sum_{i=1}^{\bar{n}} \sum_{\mu=1}^{s_{i}} \sum_{j=1}^{n}\left[\psi_{i \mu}^{(o)} \cdot \varepsilon_{i j}+\psi_{j}^{(k)}+w_{2}^{(o)} \alpha_{i \mu j}^{(o)}\right] u_{i \mu j}^{(o)}, \\
H_{2}=\sum_{i=1}^{\bar{n}} \sum_{\mu=1}^{s_{i}} \sum_{j=1}^{n}\left[\psi_{i \mu j}^{(f)}+w_{5}^{(f)} \beta_{i \mu}^{(f)}\right] u_{i \mu j}^{(f)},
\end{gathered}
$$

where $\psi(t)$ is the conjunctive vector. 
The maximisation of the Hamiltonian $H_{1}$ for model (1) in combination with the constraints (2)-(4) solves the assignment problem. The maximisation of the Hamiltonian $\mathrm{H}_{2}$ for model (13) in combination with the constraints (14)-(15) solves the LP problem. At each time instant, only those jobs and constraints from the 'active scheduling zone' (i.e. the area marked in grey in Figures 2-5) are considered in the models $\mathbf{M}_{\boldsymbol{o}}$ and $\mathbf{M}_{\boldsymbol{f}}$ which meet the requirements (2)-(4), (5), (14) and (15). By a dynamic switching of the constraints (3) from inequalities to equalities, the size of the scheduling problem at each time point is reduced. The Hamiltonians (20) and (21) can be maximised when the constraints (3) satisfy the corresponding variables $u_{i \mu j}^{(o)}$ and $u_{i \mu j}^{(f)}$. In this case, only a part of the constraints (3) and (14) is considered for the current assignment problem since, when the control in (3) is switched to zero, then it becomes active in the right-hand part of the Equations (14). Therefore, the reduction of the problem dimensionality at each time instant in the calculation process is ensured due to the recurrent operation description.

Theorem 1 Let $\Lambda$ be a relaxed problem for the problem PS. Then,

(a) If the problem $\Lambda$ does not have a feasible solution, then this is true for the problem $P S$ as well.

(b) If the OPC of the problem $\Lambda$ is feasible, then it is the OPC for the problem PS as well.

Proof

(a) If the problem $\Lambda$ does not have a feasible solution, then the control $\mathbf{u}(t)$ that transfers the dynamic systems (1)-(4) and (11), (13)-(15) $\dot{\mathbf{x}}=\mathbf{f}(\mathbf{x}, \mathbf{u}, t)$ from a given initial state to a given final state does not exist.

(b) Let $\mathbf{u}^{*}(t), \forall t \in\left(T_{0}, T_{f}\right]$, be an OPC in $\Lambda$ and $\mathbf{x}(t)$ be a solution to model $\mathbf{M}$ subject to $\mathbf{u}(t)=\mathbf{u}^{*}(t)$. Then, $\mathbf{u}^{*}(t)$ meets the requirements of the local cut method and maximises the Hamiltonian for the problem PS. Hence, the vectors $\mathbf{u}^{*}(t)$ and $\mathbf{x}^{*}(t)$ return the minimum to the performance indicators (9)-(10), (12) and (16)-(17). The proof is complete.

Corollary 1. The PS problem can be transferred to a boundary problem.

Proof. As the dynamics of the state and conjunctive variables $\mathbf{x}(t)$ and $\psi(t)$ is described by the differential equations, it becomes possible to calculate $\mathbf{x}(t)$ and $\psi(t)$ at any time instant subject to the given initial conditions $\left(x_{0}, \psi_{0}\right)$. The Hamiltonian (19) turns into a function of only one variable $\mathbf{u}(t)$ that can be calculated at any $t$ subject to $\mathbf{u}(t) \in Q_{\mathbf{u}}$. Therefore, the PS problem can be reduced to a two-point boundary problem with the help of the local cut method (Boltyanskiy 1973). The proof is complete.

A methodical challenge in applying the maximum principle is to find the coefficients of the conjunctive system which change in dynamics. One of the contributions of this study is that these coefficients can be found analytically from Equations (23) to (24). The coefficients of the conjunctive system play the role of the dynamical Lagrange multipliers as compared with MP dual formulations.

The conjugate system can be written as follows (Boltyanskiy 1973; Moiseev 1974):

$$
\dot{\psi}_{\tilde{l}}=-\frac{\partial H}{\partial x_{\tilde{l}}}+\sum_{\tilde{\alpha}=1}^{\tilde{I}_{1}} \delta_{\tilde{\alpha}}(t) \frac{\partial \mathbf{q}_{\tilde{\alpha}}^{(1)}(\mathbf{x}(t), \mathbf{u}(t))}{\partial x_{\tilde{l}}}+\sum_{\tilde{\beta}=1}^{\tilde{I}_{2}} \rho_{\tilde{\beta}}(t) \frac{\partial \mathbf{q}_{\beta}^{(2)}(\mathbf{x}(t), \mathbf{u}(t))}{\partial x_{\tilde{l}}}
$$

The coefficients $\delta_{\tilde{\alpha}}(t), \rho_{\tilde{\beta}}(t)$ can be determined by means of the following expressions (23)-(24):

$$
\begin{gathered}
\rho_{\tilde{\beta}}(t) \mathbf{q}_{\tilde{\beta}}^{(2)}(\mathbf{x}(t), \mathbf{u}(t)) \equiv 0, \tilde{\beta} \in\left\{1, \ldots, \tilde{I}_{2}\right\} \\
\operatorname{grad}_{\mathbf{u}} H(\mathbf{x}(t), \mathbf{u}(t), \psi(t))=\sum_{\tilde{\alpha}=1}^{\tilde{I}_{1}} \delta_{\tilde{\alpha}}(t) \operatorname{grad}_{\mathbf{u}} \mathbf{q}_{\tilde{\alpha}}^{(1)}(\mathbf{x}(t), \mathbf{u}(t))+\sum_{\tilde{\beta}=1}^{\tilde{I}_{2}} \rho_{\tilde{\beta}}(t) \operatorname{grad}_{\mathbf{u}} \mathbf{q}_{\tilde{\beta}}^{(2)}(\mathbf{x}(t), \mathbf{u}(t)) .
\end{gathered}
$$

In the formulas (22)-(24), $x_{\tilde{\tilde{I}}}$ are the elements of the general state vector $\mathbf{x}(t)$ and $\boldsymbol{\psi}(t)$ are the elements of the conjugate vector $\psi(t)$. In accordance with the maximum principle, the following conjugate system can be written (25)-(27):

$$
\begin{gathered}
\frac{d \psi_{i \mu}^{(o)}}{d t}=\dot{\psi}_{i \mu}^{(o)}=-\sum_{j=1}^{n}\left[\psi_{i(\mu+1)}^{(o)} \varepsilon_{i j}+\psi_{j}^{(k)}+\lambda_{2}^{(o)} \alpha_{i(\mu+1) j}^{(o)}\right] u_{i(\mu+1) j}^{(o)}, \\
\frac{d \psi_{j}^{(k)}}{d t}=\dot{\psi}_{j}^{(k)}=0 \\
\frac{d \psi_{i \mu j}^{(f)}}{d t}=\dot{\psi}_{i \mu j}^{(f)}=0
\end{gathered}
$$


The transversality conditions can be formulated in the following way (28)-(30):

$$
\begin{gathered}
\psi_{i \mu}^{(o)}\left(T_{f}\right)=\lambda_{1}^{(o)}\left(a_{i \mu}^{(o)}-x_{i \mu}^{(o)}\left(T_{f}\right)\right) ; \\
\psi_{j}^{(k)}\left(T_{f}\right)=\lambda_{3}^{(k)}\left(T-x_{j}^{(k)}\left(T_{f}\right)\right) ; \\
\psi_{i \mu j}^{(f)}\left(T_{f}\right)=\lambda_{5}^{(f)}\left(a_{i \mu j}^{(f)}-x_{i \mu j}^{(f)}\left(T_{f}\right)\right) ;
\end{gathered}
$$

Let us consider the algorithmic realisation of the above-described modified maximum principle. After transforming into a boundary problem, a relaxed problem can be solved to receive an OPC, for the computation of which the main and conjunctive systems are integrated, that is the OPC vector $\mathbf{u}^{*}(t)$ and the state trajectory $\mathbf{x}^{*}(t)$ are obtained. The OPC vector at time $t=T_{0}$ and for the given value of $\psi(t)$ should return the maximum to (9)-(10), (12) and (16)-(17), which have been transformed to a general performance index and expressed in scalar form $J_{G}$.

The basic peculiarity of the boundary problem considered is that the initial conditions for the conjunctive variables $\boldsymbol{\psi}\left(t_{0}\right)$ are not given. At the same time, an OPC should be calculated subject to the end conditions (5)-(8). To obtain the conjunctive system vector, we use the Krylov-Chernousko method of successive approximations (MSA) for an OPC problem with a free right end which is based on the joint use of a modified successive approximation method (Krylov and Chernousko 1972). We propose to use a heuristic schedule $\overline{\mathbf{u}}(t)$ to obtain the initial conditions for $\psi\left(t_{0}\right)$. Then, the algorithm DYN can be stated as follows:

Step 1 An initial solution $\overline{\mathbf{u}}(t), t \in\left(T_{0}, T_{f}\right]$ (a feasible control, in other words, a feasible schedule) is selected and $r=0$.

Step 2 As a result of the dynamic model run, $\mathbf{x}^{(r)}(t)$ is received. Besides, if $t=T_{f}$, then the record value $J_{G}=J_{G}^{(r)}$ can be calculated. Then, the transversality conditions (30)-(32) are evaluated.

Step 3 The conjugate system (27)-(29) is integrated subject to $\mathbf{u}(t)=\overline{\mathbf{u}}(t)$ and over the interval from $t=T_{f}$ to $t=T_{0}$. For the time $t=T_{0}$, the first approximation $\psi_{i}^{(r)}\left(T_{0}\right)$ is obtained as a result. Here, the iteration number $r=0$ is completed.

Step 4 From the time point $t=T_{0}$ onwards, the control $\mathbf{u}^{(r+1)}(t)$ is determined $(r=0,1,2, \ldots$ denotes the number of the iteration). In parallel with the maximisation of the Hamiltonian, the main system of equations and the conjugate one are integrated. The maximisation involves the solution of several MP problems at each time point.

The assignments (i.e. the control variables $u_{i \mu j}^{(o)}$ ) from the model $\mathbf{M}_{o}$ are used in the flow control $\mathbf{M}_{\boldsymbol{f}}$ (13)-(17) by means of the constraints (14). At the same time, the model $\mathbf{M}_{f}$ influences the model $\mathbf{M}_{\boldsymbol{o}}$ through the transversality conditions (28)-(30), the conjunctive system (25)-(27) and the Hamiltonian function (19). In addition, the possible machine structure dynamics and flow control dynamics through perturbation impacts are taken into account in (11) and (15).

In each iteration, the main part of the functional $J_{G}$ is negative and has a maximal absolute value among the main parts of increments computed for all possible variations of the control variables. In contrast to gradient methods and classical formulas of calculus of variations, it is sufficient to use a smallness of the integral increment norm $\|\delta \mathbf{u}\|$ for decreasing $J_{G}$. The smallness $|\delta \mathbf{u}|$ at the planning interval $\sigma=\left(T_{o}, T_{f}\right]$ is not necessary. The advantage of MSA is that it allows to implement needle control variations subject to the whole area of feasible control actions subject to the given constraint system, that is the area of feasible schedules. Another MSA advantage is that the search for an optimal control in each iteration is performed in the class of boundary (e.g. pointwise or relay) functions which correspond to the discrete nature of decision-making in scheduling.

Note that the MSA in its initial form has not guaranteed the convergence. By now, a number of MSA modifications with proved convergence exist (Lyubushin 1979). The following MSA modification can be used for the considered scheduling algorithm. In step 4, formula (31) is used for the maximisation of the Hamiltonian:

$$
\vec{u}^{(r+1)}(t)=\left\{\begin{array}{c}
R u^{(r)}(t), t \in\left[t^{\prime}, t^{\prime \prime}\right] \\
u^{(r)}(t), t \notin\left[t^{\prime}, t^{\prime \prime}\right]
\end{array},\right.
$$

where $\left[t^{\prime}, t^{\prime \prime}\right]$ is selected so that a decrease

$$
J_{G}^{(r+1)}<J_{G}^{(r)}
$$

is ensured.

$J_{G}^{(r)}, J_{G}^{(r+1)}$ are the values of the goal functional subject to $\mathbf{u}^{(r)}, \mathbf{u}^{(r+1)}$, respectively. In Equation (31), the operator $R$ corresponds to step 4 of the DYN algorithm. The selection of $t^{\prime}, t^{\prime \prime}$ in each iteration is based on the values of the Hamiltonian functions of two subsequent iterations. An example is provided in Figure 7. 


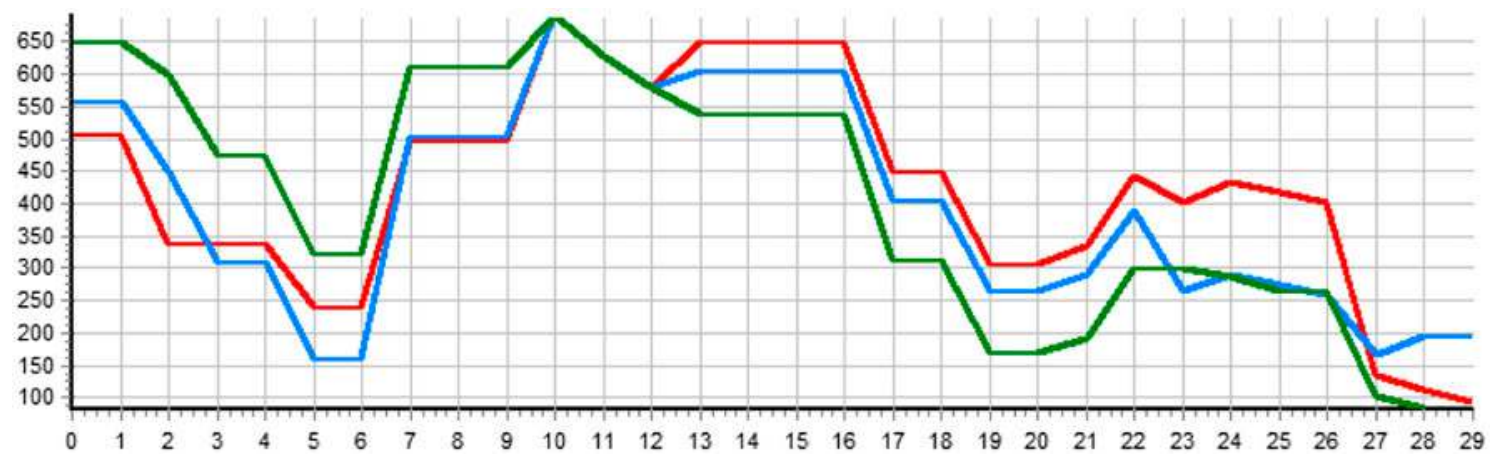

Figure 7. Hamiltonian function values in different iterations.

At the $Y$-axis, the values of the Hamiltonian function (19) are presented. The $X$-axis represents the instants of the time interval $[0,29]$. The three lines correspond to three computational iterations and depict the values of the Hamiltonian function (19) at different time instants. The red line (starting at $t=0$ with the value 500) refers to the first iteration; the blue line (starting at $t=0$ with the value 550) refers to the second iteration; and the green line (starting at $t=0$ with the value 650) refers to the third iteration. It can be observed that within $t \in[0,12]$, the schedule calculated at the third iteration provides better results. For instance, $\mathrm{H}_{1}$ (Equation 20) increases in the case of lower penalties. Similarly, $\mathrm{H}_{2}$ (Equation 21) increases in the case of higher values of $u_{i \mu j}^{(f)}$ which means a higher volume of processed goods.

However, within $t \in[12,27]$, the first scheduling iteration outperforms the other iterations. For $t \in[27,29]$, the third iteration does not improve the results of the second iteration, that is the schedule of the second iteration for $t \in[27,29]$ shall be used. In combining the upper bounds of the Hamiltonian functions, an optimal schedule is calculated. It contains, for this example, the results of the third iteration (green line) for $t \in[0,12]$, the first iteration (red line) for $t \in[12,27]$ and the second iteration (blue line) for $t \in[27,29]$.

The dynamic coordination parameters are conjunctive variables which change their values during the iterative process of the corresponding two-point boundary problem solution. At each time instant, the global objective function is the Hamiltonian (19). The locally coordinated sub-problems are partial combinatorial assignment problems (Equations (20), (2)-(4)) and LP problems (Equations (21), (14)-(15)) which are formed dynamically in dependence on the current active scheduling zone (see Figures 2-5).

If so, the values of the conjunctive variables (i.e. the coordinated signals) change from one iteration to the next iteration and can be considered in some sense as a generalisation of the coordination parameters previously considered in the decomposition-coordination procedures of Dantzig and Wolfe (1960) (goal coordination) and Kornai and Lipták (1965) (resource coordination). In the proposed approach, the conjunctive variables and the Hamiltonian function allow at each time instant to perform both the goal and the resource coordination of the solutions which are found in the combinatorial sub-problems.

In addition, the developed modification of the MSA method guarantees a monotonic change of the conjunctive variable values by both the transversality conditions (28)-(30) and a situational selection of the Hamiltonian function values. The transversality conditions interconnect the state parameter values in the main and conjunctive systems (see Equations (1), (11), (13), (25)-(27)) at the time moment that corresponds to the end of the planning interval. The values of the Hamiltonian function (19) are saved during the iterative search procedure (see Figure 7). The theoretical convergence of the considered iterative procedure has been previously proved in Lyubushin (1979) and Chernousko and Lyubushin (1982).

\section{Optimality and complexity analysis}

In this section, we analyse the model and the algorithm from Sections 4 and 5.

\subsection{Optimality and existence analysis}

Proposition 1 The optimal programme control $\mathbf{u}(t)$ is an optimal flow-shop schedule.

Existence proof. The formulated model is a linear non-stationary finite-dimensional control system of differential equations with a convex area of feasible control. This model form satisfies the conditions of the existence theorem in 
Lee and Markus 1967, Theorem 4, Corollary 2), which allows us to assert the existence of an optimal solution in the appropriate class of feasible controls. According to Lee and Markus (1967, Theorem 4, Corollary 2), along with the initial class $\tilde{K}$ formed via the constraints $\mathbf{q}^{(1)}$ and $\mathbf{q}^{(2)}$ describing the domain $\mathbf{Q}(\mathbf{x}(t))$, an extended class $\tilde{\tilde{K}}$ of control inputs can be considered. In the extended class $\tilde{\tilde{K}}$, the relay constraints $u_{i \mu j}^{(o)}(t) \in\{0 ; 1\}$ are substituted by a less strict one $u_{i \mu j}^{(o)}(t) \in[0 ; 1]$ (u is substituted by $\tilde{\tilde{\mathbf{u}}}$ ). In this case, an extended domain $\tilde{\tilde{\mathbf{Q}}}(\mathbf{x}(t))$ of feasible control inputs may be formed by means of special transformations ensuring the convexity and the compactness of $\mathbf{Q}(\mathbf{x}(t))$ (Moiseev 1974). The theorem of Lee and Markus (1967) confirms that all the conditions for the existence of an optimal control for the extended control class $\tilde{\tilde{K}}$ are valid. If in a given class of feasible control actions $\tilde{\tilde{K}}$, an optimal control $\tilde{\tilde{\mathbf{u}}}(t)$ exists, then, as arises from the local cut method, the control $\tilde{\tilde{\mathbf{u}}}(t)$ returns at each time instant $t \in\left(T_{0}, T_{f}\right]$ at the set $\tilde{\tilde{\mathbf{Q}}}(\mathbf{x}(t))$ the maximum to the Hamiltonian (19)-(21). The proof is complete.

Optimality proof. An analysis of (19)-(21) shows that the Hamiltonian is linear in $\tilde{\tilde{\mathbf{u}}}$. Since $\tilde{\tilde{\mathbf{Q}}}(\mathbf{x}(t))$ is a linear capsule of $\mathbf{Q}(\mathbf{x}(t))$, the maximisation of the Hamiltonian (19)-(21) over the sets $\mathbf{Q}$ and $\tilde{\mathbf{Q}}$ leads to the same results. An optimal control for the class $\tilde{K}$ belongs to the class $\tilde{\tilde{K}}$. Taking into account $\tilde{K} \subset \tilde{K}$, this control is also optimal for the class $\tilde{K}$. Therefore, the relaxed problem can be solved instead of the initial one to obtain an optimal feasible control for the class $\tilde{K}$. The proof is complete.

Corollary 2 An analysis of the studies (Boltyanskiy 1973; Moiseev 1974) shows that for a linear non-stationary finite-dimensional system (model M) with a convex area of an feasible control $Q(\boldsymbol{x})$ and the goal vector, the stated necessary optimality conditions are also sufficient conditions.

\subsection{Analysis of the complexity of the algorithm}

It is known from the literature that the MSA method can be easily implemented when programming an algorithm. It also allows a fast computation. One MSA iteration corresponds to one iteration of a gradient method. The MSA differs from the gradient methods in calculating the Hamiltonian function (19) in each iteration with the usage of the previous iteration data.

Proposition 2 The computational complexity of the MSA in one iteration is polynomial. With regard to the considered scheduling problem, the complexity of the proposed algorithm in one iteration is determined by the complexity of the IP assignment problem (20), (2)-(4) and the LP problem (21), (14)-(15).

Proof On the basis of the works on the Hungarian method, the complexity of the IP problem can be estimated according to the formula

$$
O\left(\overline{\bar{m}}_{1}^{3}\right)\left(\frac{\sigma}{\Delta t}\right)
$$

where $O\left(\overline{\bar{m}}_{1}\right)=\frac{11 \overline{\bar{m}}_{1}^{3}+12 \overline{\bar{m}}_{1}^{2}+3 \bar{m}_{1}}{6}$ is the maximal number of the ' + ' operations at one time point of the planning period $\left(T_{o}, T_{f}\right]$ for the assignment problem being solved via the Hungarian method;

$\overline{\bar{m}}_{1}$ is the maximal dimensionality of the assignment problem, that is the number of independent paths in the schedule execution network diagram. For example, in Figures $2-5$, the dimensionality is equal to the job number $\bar{B}^{(i)}$, that is $\bar{n}=6$;

$\sigma$ is the duration of the planning interval;

$\Delta t$ is the step length of integration for the main and the conjugate system.

Note that the integration of the differential equations in the main and the conjugate system is not necessary due to their linearity. The computation can be performed subject to simple recurrent formulas. The step for the recurrent calculations may be variable, subject to significant events which influence the current («active") field of operations (grey coloured operations in Figures 2-5). These events may include, for example the completion of one operation, a pre-emption of an operation or an arrival of a new operation.

Complexity of the LP problem (21), (14)-(15) can be estimated according to the complexity of the simplex method which can be used to solve the above-mentioned LP problem. This complexity can be estimated for one iteration as $O\left((\tilde{\bar{n}} \cdot \bar{s} \cdot n)^{2}+\tilde{\bar{n}} \cdot \bar{s} \cdot n^{2}\right)$, where $\tilde{\bar{n}}$ is the maximal number of jobs executed in parallel within the planning interval, $\bar{s}$ is the average operation number of the jobs and $n$ is the maximal number of machines working in parallel. Similar to (33), the complexity of the LP calculation within the MSA can be estimated as follows:

$$
O\left((\tilde{\bar{n}} \cdot \bar{s} \cdot n)^{2}+\tilde{\bar{n}} \cdot \bar{s} \cdot n^{2}\right) \cdot\left(\frac{\sigma}{\Delta t}\right)
$$


Thus, the total complexity at one MSA iteration is as follows:

$$
\overline{\bar{N}}<\left[O\left(\overline{\bar{m}}_{1}\right)+O\left((\tilde{\bar{n}} \cdot \bar{s} \cdot n)^{2}+\tilde{\bar{n}} \cdot \bar{s} \cdot n^{2}\right)\right] \cdot\left(\frac{\sigma}{\Delta t}\right),
$$

where $\overline{\bar{N}}$ is the average number of addition operations.

Since the complexity of the IP/LP problem at each cut is polynomial and the number of integration steps and iterations increases linearly, the computational complexity of the DYN algorithm is polynomial. The proof is complete.

\section{Conclusions}

In this study, we considered non-deterministic issues in flow-shop scheduling where scheduling is interconnected to the control function. We included such factors like temporal unavailability of machines and fluctuations of processing times and technological constraints. This forms a dynamic scheduling environment which is important for short-term scheduling in smart factories Industry 4.0.

The proposed dynamic decomposition is supported both with an algorithm of local coordination with the help of MP (i.e. at each time instant) and an algorithm of global optimisation (i.e. upon the whole planning horizon). This results in the formulation and solution of partial combinatorial problems of lower dimensionality.

The proposed modification of the classical OPC model helps to update (e.g. due to dynamic changes in capacity availability) nonlinear constraints on a convex domain of feasible control inputs rather than in the right-hand sides of differential equations. The coefficients of the conjunctive system (i.e. the dynamic Lagrange coefficients) keep the information about the operational and logical constraints and can be explicitly defined via the local cut method (Boltyanskiy 1973).

Furthermore, we proposed to substitute the relay constraints by interval ones, that is instead of the relay constraints $\mathbf{u}_{i \mu j} \in\{0,1\}$ less strict ones $\mathbf{u}_{i \mu j} \in[0,1]$ can be considered. The control takes Boolean values as it is caused by the linearity of the differential equations and the convexity. The proposed substitution enables us to use fundamental scientific results of the OPC theory in scheduling.

In light of this result, the contribution of this study is directed towards a theoretical formulation of a practically important scheduling problem for a new smarty factory domain with the help of a sophisticated scientific methodology.

Let us analyse some limitations and future research work. Since the DYN algorithm is launched by heuristics procedures, the performance of these heuristics may be compared with each other and with the optimal DYN solution. This research has not intended so far to outperform the heuristics or MP algorithms but rather extends them. This is the focus of our future efforts.

\section{Acknowledgement}

The authors thank the anonymous referees for their valuable comments and improvement suggestions.

\section{Funding}

The research described in this paper is partially supported by the Russian Foundation for Basic Research [15-07-08391, 15-08-08459, 13-07-00279, 13-08-01250, 13-07-12120, 13-07-00279]; [grant 074-U01] supported by Government of Russian Federation; Department of nanotechnologies and information technologies of the RAS (project 2.11), and project "5-100-2020" (arrangement 6.1.1); supported by NRU St. Petersburg SPU.

\section{References}

Athaus, M., and P. L. Falb. 1966. Optimal Control: An Introduction to the Theory and Its Applications. New York: McGraw-Hill. Blazewicz, J., M. Dror, and J. Weglarz. 1991. "Mathematical Programming Formulations for Machine Scheduling: A Survey." European Journal of Operational Research 51: 283-300.

Blazewicz, J., K. Ecker, E. Pesch, G. Schmidt, and J. Weglarz. 2001. Scheduling Computer and Manufacturing Processes. 2nd ed. Berlin: Springer Verlag.

Boltyanskiy, B. 1973. Optimal Control of Discrete Systems. Moscow: Nauka.

Bożek, A., and M. Wysocki. 2015. "Flexible Job Shop with Continuous Material Flow." International Journal of Production Research 53 (4): 1273-1290.

Camarinha-Matos, L. M., and P. Macedo. 2010. “A Conceptual Model of Value Systems in Collaborative Networks.” Journal of Intelligent Manufacturing 21 (3): 287-299. 
Chernousko, F. L., and A. A. Lyubushin. 1982. "Method of Successive Approximations for Solution of Optimal Control Problems." Optimal Control Applications and Methods 3 (2): 101-114.

Chick, S. E., A. Huchzermeier, and S. Netessine. 2014. Europe's Solution Factories. Harvard Business Review, April 2014.

Chiou, C. W., W. M. Chen, C. M. Liu, and M. C. Wu. 2012. "A Genetic Algorithm for Scheduling Dual Flow Shops.” Expert Systems with Applications 39 (1): 1306-1314.

Dantzig, G. B., and P. Wolfe. 1960. "Decomposition Principle for Linear Programs.” Operations Research 8: $101-111$.

Davis, J., T. Edgar, J. Porter, J. Bernaden, and M. Sarli. 2012. "Smart Manufacturing, Manufacturing Intelligence and Demanddynamic Performance." Computers \& Chemical Engineering 47: 145-156.

Dolgui, A., and J.-M. Proth. 2010. Supply Chains Engineering: Useful Methods and Techniques. Berlin: Springer.

Dugardin, F., F. Yalaoui, and L. Amodeo. 2010. "New Multi-objective Method to Solve Reentrant Hybrid Flow Shop Scheduling Problem.” European Journal of Operational Research 203 (1): 22-31.

Gonzalez, T., and S. Sahni. 1978. "Flowshop and Jobshop Schedules: Complexity and Approximation." Operations Research 26: $36-52$.

Graham, R. L., E. L. Lawler, J. K. Lenstra, and A. H. G. Rinnooy Kan. 1979. "Optimization and Approximation in Deterministic Sequencing and Scheduling Theory: A Survey." Annals of Discrete Mathematics 5: 287-326.

Gupta, J. N. D., V. R. Neppalli, and F. Werner. 2001. "Minimizing Total Flow Time in a Two-machine Flowshop Problem with Minimum Makespan." International Journal of Production Economics 69: 323-338.

Hankins, S. L., R. A. Wvsk, and K. R. Fox. 1984. "Using a CATS Database for Alternative Machine Loading." Journal of Manufacturing Systems 3: 115-120.

Harjunkoski, I., C. T. Maravelias, P. Bongers, P. M. Castro, S. Engell, I. E. Grossmann, J. Hooker, C. Méndez, G. Sand, and J. Wassick. 2014. "Scope for Industrial Applications of Production Scheduling Models and Solution Methods." Computers \& Chemical Engineering 62: 161-193.

Holt, C. C., F. Modigliani, J. F. Muth, and H. A. Simon. 1960. Planning Production, Inventories and Work Force. Englewood Cliffs, NJ: Prentice Hall.

Hwang, C. L., L. T. Fan, and L. E. Erikson. 1967. “Optimum Production Planning by the Maximum Principle.” Management Science 13 (9): 751-755.

Ivanov, D., and B. Sokolov. 2012a. "Dynamic Supply Chain Scheduling.” Journal of Scheduling 15 (2): 201-216.

Ivanov, D., and B. Sokolov. 2012b. "Structure Dynamics Control Approach to Supply Chain Planning and Adaptation." International Journal of Production Research 50 (21): 6133-6149.

Ivanov, D., B. Sokolov, and J. Kaeschel. 2010. "A Multi-structural Framework for Adaptive Supply Chain Planning and Operations Control with Structure Dynamics Considerations.” European Journal of Operational Research 200: 409-420.

Ivanov, D., B. Sokolov, and A. Pavlov. 2013. "Dual Problem Formulation and Its Application to Optimal Redesign of an Integrated Production-Distribution Network with Structure Dynamics and Ripple Effect Considerations.” International Journal of Production Research 51 (18): 5386-5403.

Ivanov, D., B. Sokolov, and A. Dolgui. 2014. "The Ripple Effect in Supply Chains: Trade-off 'Efficiency-Flexibility-Resilience' in Disruption Management.” International Journal of Production Research 52 (7): 2154-2172.

Ivanov, D., B. Sokolov, and A. Pavlov. 2014. "Optimal Distribution (Re)Planning in a Centralized Multi-stage Supply Network under Conditions of the Ripple Effect and Structure Dynamics.” European Journal of Operational Research 237 (2): $758-770$.

Ivanov, D., B. Sokolov, and E. A. Dilou Raguinia. 2014. "Integrated Dynamic Scheduling of Material Flows and Distributed Information Services in Collaborative Cyber-physical Supply Networks." International Journal of Systems Science: Operations \& Logistics 1 (1): 18-26.

Johnson, S. 1954. "Optimal Two- and Three-stage Production Schedules with Setup times Included.” Naval Research Logistics Quarterly 1: 61-68.

Józefowska, J., M. K. Mika, R. Różycki, G. Waligóra, and J. Węglarz. 2002. “A Heuristic Approach to Allocating the Continuous Resource in Discrete-Continuous Scheduling Problems to Minimize the Makespan.” Journal of Scheduling 5 (6): $487-499$.

Jungwattanakit, J., M. Reodecha, P. Chaovalitwongse, and F. Werner. 2008. "Algorithms for Flexible Flow Shop Problems with Unrelated Parallel Machines, Setup times, and Dual Criteria." The International Journal of Advanced Manufacturing Technology 37: 354-370.

Jungwattanakit, J., M. Reodecha, P. Chaovalitwongse, and F. Werner. 2009. "A Comparison of Scheduling Algorithms for Flexible Flow Shop Problems with Unrelated Parallel Machines, Setup Times, and Dual Criteria." Computers \& Operations Research 36 (2): $358-378$.

Khmelnitsky, E., K. Kogan, and O. Maimom. 1997. "Maximum Principle-based Methods for Production Scheduling with Partially Sequence-dependent Setups.” International Journal of Production Research 35 (10): 2701-2712.

Kimemia, J. G., and T. I. Gershwin. 1983. "An Algorithm for the Computer Control of a Flexible Manufacturing System." IIE Transactions 15: 353-362.

Kornai, J., and T. Lipták. 1965. “Two-level Planning.” Econometrica 33: 141-169.

Krylov, I. A., and F. L. Chernousko. 1972. "An Algorithm for the Method of Successive Approximations in Optimal Control Problems." Zhurnal Vychislitel'noi Matematiki i Matematicheskoi Fiziki 12 (1): 14-34. 
Kubzin, M. A., C. N. Potts, and V. A. Strusevich. 2009. “Approximation Results for Flow Shop Scheduling Problems with Machine Availability Constraints." Computers \& Operations Research 36 (2): 379-390.

Kyparisis, G. J., and C. P. Koulamas. 2006. "Flexible Flow Shop Scheduling with Uniform Parallel Machines.” European Journal of Operational Research 168: 985-997.

Laha, D., and S. C. Sarin. 2009. “A Heuristic to Minimize Total Flow Time in Permutation Flow Shopł.” Omega 37 (3): $734-739$.

Lauff, V., and F. Werner. 2004. "On the Complexity and Some Properties of Multi-stage Scheduling Problems with Earliness and Tardiness Penalties." Computers \& Operations Research 31: 317-345.

Lee, E. B., and L. Markus. 1967. Foundations of Optimal Control Theory. New York: Wiley.

Lyubushin, A. A. 1979. "Modifications and Convergence of Successive Approximations for Optimal Control Problems." Zhurnal Vychislitel'noi Matematiki i Matematicheskoi Fiziki 19 (6): 1414-1421.

Maccarthy, B. L., and J. Liu. 1993. "Addressing the Gap in Scheduling Research: A Review of Optimization and Heuristic Methods in Production Scheduling." International Journal of Production Research 31 (1): 59-79.

Martin, C. H. 2009. "A Hybrid Genetic Algorithm/Mathematical Programming Approach to the Multi-family Flowshop Scheduling Problem with Lot Streaming tै." Omega 37 (1): 126-137.

Moiseev, N. N. 1974. Element of the Optimal Systems Theory [in Russian]. Moscow: Nauka.

Mujawar, S., S. Huang, and R. Nagi. 2012. "Scheduling to Minimize Stringer Utilization for Continuous Annealing Operations." Omega 40 (4): 437-444.

Na, H., and J. Park. 2013. "Multi-level Job Scheduling in a Flexible Job Shop Environment." International Journal of Production Research 52 (13): 3877-3887.

Pinedo, M. L. 2008. Scheduling: Theory, Algorithms, and Systems. New York: Springer.

Pontryagin, L. S., V. G. Boltyanskiy, R. V. Gamkrelidze, and E. F. Mishchenko. 1964. The Mathematical Theory of Optimal Processes. Oxford: Pergamon Press.

PWC. 2013. Deutschland Hinkt Bei Industrie 4.0 Hinterher - Smart Factory' Etabliert Sich Nur Langsam. Accessed August 28, 2014. http://www.pwc.de/de/pressemitteilungen/2013/deutschland-hinkt-bei-industrie-4-0-hinterher-smart-factory-etabliert-sichnur-langsam.jhtml

Rajabinasab, A., and S. Mansour. 2011. "Dynamic Flexible Job Shop Scheduling with Alternative Process Plans: An Agent-based Approach." The International Journal of Advanced Manufacturing Technology 54 (9-12): 1091-1107.

Ribas, I., R. Leisten, and J. M. Framiñan. 2010. "Review and Classification of Hybrid Flow Shop Scheduling Problems from a Production System and a Solutions Procedure Perspective." Computers \& Operations Research 37 (8): 1439-1454.

Sarimveis, H., P. Patrinos, C. D. Tarantilis, and C. T. Kiranoudis. 2008. "Dynamic Modeling and Control of Supply Chain Systems: A Review." Computers \& Operations Research 35: 3530-3561.

Sawik, T. 2012. "Batch Versus Cyclic Scheduling of Flexible Flow Shops by Mixed-integer Programming." International Journal of Production Research 50 (18): 5017-5034.

Sethi, S. P., and G. L. Thompson. 2000. Optimal Control Theory: Applications to Management Science and Economics. 2nd ed. Berlin: Springer.

Shah, N. K., and M. G. Ierapetritou. 2012. "Integrated Production Planning and Scheduling Optimization of Multisite, Multiproduct Process Industry." Computers \& Chemical Engineering 37 (10): 214-226.

Subramanian, K., J. B. Rawlings, C. T. Maravelias, J. Flores-Cerrillo, and L. Megan. 2013. "Integration of Control Theory and Scheduling Methods for Supply Chain Management." Computers \& Chemical Engineering 51: 4-20.

Tahar, D. N., F. Yalaoui, C. Chu, and L. Amodeo. 2006. "A Linear Programming Approach for Identical Parallel Machine Scheduling with Job Splitting and Sequence-dependent Setup Times." International Journal of Production Economics 99 (1-2): 63-73.

Villa, A., C. O’Brien, and P. Burlat. 2012. "Editorial: Supply Networks Design and Management." Journal of Intelligent Manufacturing 23 (6): 2441-2442.

Weglarz, J. 1976. "Time-optimal Control of Resource Allocation in a Complex-of-operations Framework." IEEE Transactions on Systems, Man and Cybernetics SMC-6/11, 783-788.

Werner, F. 2013. "A Survey of Genetic Algorithms for Shop Scheduling Problems." In Heuristics: Theory and Applications, edited by P. Siarry, 161-222. Hauppauge, NY: Nova Science Publisher.

Yu, W., Z. Liu, L. Wang, and T. Fan. 2011. "Routing Open Shop and Flow Shop Scheduling Problems." European Journal of Operational Research 213 (1): 24-36.

Zhuge, H. 2011. "Semantic Linking through Spaces for Cyber-Physical-Socio Intelligence: A Methodology." Artificial Intelligence 175 (5-6): 988-1019.

Zimin, I. N., and Yu. P. Ivanilov. 1971. "Solution of Network Planning Problems by Reducing Them to Optimal Control Problems." Zhurnal Vychislitel'noi Matematiki i Matematicheskoi Fiziki 11 (3): 632-641. 